\title{
Adolescent cocaine exposure simplifies orbitofrontal cortical dendritic arbors
}

\author{
Lauren M. DePoy 1,2,3, Riley E. Perszyk ${ }^{4}$, Kelsey S. Zimmermann ${ }^{1,2,3}$, Anthony J. Koleske ${ }^{5,6,7}$ and \\ Shannon L. Gourley ${ }^{1,2,3 *}$
}

1 Department of Pediatrics, Emory University School of Medicine, Atlanta, GA, USA

2 Yerkes National Primate Research Center, Emory University, Atlanta, GA, USA

${ }^{3}$ Graduate Program in Neuroscience, Emory University, Atlanta, GA, USA

${ }^{4}$ Graduate Program in Molecular and Systems Pharmacology, Emory University, Atlanta, GA, USA

${ }^{5}$ Department of Molecular Biophysics and Biochemistry, Yale University, New Haven, CT, USA

${ }^{6}$ Interdepartmental Neuroscience Program, Yale University, New Haven, CT, USA

${ }^{7}$ Department of Neurobiology, Yale University School of Medicine, New Haven, CT, USA

\section{Edited by:}

M. Foster Olive, Arizona State University, USA

\section{Reviewed by:}

Olivier George, The Scripps

Research Institute, USA

Lynn G. Kirby, Temple University

School of Medicine, USA

${ }^{*}$ Correspondence:

Shannon L. Gourley, Yerkes National Primate Research Center, Emory University, 954 Gatewood Road NE, Atlanta, GA 30329, USA

e-mail:

shannon.I.gourley@emory.edu
Cocaine and amphetamine remodel dendritic spines within discrete cortico-limbic brain structures including the orbitofrontal cortex (OPFC). Whether dendrite structure is similarly affected, and whether pre-existing cellular characteristics influence behavioral vulnerabilities to drugs of abuse, remain unclear. Animal models provide an ideal venue to address these issues because neurobehavioral phenotypes can be defined both before, and following, drug exposure. We exposed mice to cocaine from postnatal days 31-35, corresponding to early adolescence, using a dosing protocol that causes impairments in an instrumental reversal task in adulthood. We then imaged and reconstructed excitatory neurons in deep-layer oPFC. Prior cocaine exposure shortened and simplified arbors, particularly in the basal region. Next, we imaged and reconstructed orbital neurons in a developmental-genetic model of cocaine vulnerability-the p190rhogap+/- mouse. p190RhoGAP is an actin cytoskeleton regulatory protein that stabilizes dendrites and dendritic spines, and p190rhogap+/- mice develop rapid and robust locomotor activation in response to cocaine. Despite this, OPFC dendritic arbors were intact in drug-naïve p190rhogap+/- mice. Together, these findings provide evidence that adolescent cocaine exposure has long-term effects on dendrite structure in the oPFC, and they suggest that cocaine-induced modifications in dendrite structure may contribute to the behavioral effects of cocaine more so than pre-existing structural abnormalities in this cell population.

Keywords: psychostimulant, orbital, sholl, adolescence, addiction

\section{INTRODUCTION}

Cocaine addiction is characterized by maladaptive decision making, a loss of control over drug consumption, and habit-like drug seeking despite adverse consequences. These cognitive changes likely reflect the effects of repeated drug exposure on prefrontal cortical neurobiology that then further promote drug use (Jentsch and Taylor, 1999; Everitt and Robbins, 2005; Torregrossa et al., 2011; Lucantonio et al., 2012). Additionally, pre-existing neurobehavioral characteristics in drug-naïve individuals may contribute to drug vulnerabilities (Ersche et al., 2012). Rodents provide an ideal model system to isolate vulnerability factors in drug-naïve organisms and also characterize the consequences of cocaine exposure because like humans, rodents will readily self-administer cocaine and engage in complex decision making, as well as relapse-like behavior. Also as in humans, individual differences in behavioral response strategies can serve as phenotypic predictors of addiction-like behaviors such as drug seeking following periods of abstinence (Deroche-Gamonet et al., 2004). Finally, even experimenter-administered, rather than self-administered, cocaine can induce behavioral phenotypes in rodents that are relevant to addiction etiology in humans, e.g., increased propensity to engage in reward-seeking habits (Schoenbaum and Setlow, 2005; Gourley et al., 2013c; Hinton et al., 2014).

We recently sought to identify the long-term consequences of cocaine exposure during adolescence, when drug use is often initiated in humans, and when the prefrontal cortex is still developing (Casey et al., 2000; Giedd, 2004; Paus et al., 2008). We found that $>7$ weeks following subchronic exposure during adolescence, cocaine-exposed mice preferentially engaged habit-like response strategies at the expense of flexible action-outcome-based strategies to acquire food reinforcers (Hinton et al., 2014). Inactivation of the orbitofrontal cortex (oPFC) blocks goal-directed action selection in the same task (Gourley et al., 2013a), and adolescent cocaine exposure eliminates dendritic spines in this region (Gourley et al., 2012). Whether adolescent cocaine exposure regulates oPFC dendrite arbor structure is, to our knowledge, unresolved. 
Such gross remodeling could contribute to persistent maladaptive decision making following adolescent cocaine exposure and potentially to increased risk of dependence (O'Brien and Anthony, 2005) or decreased likelihood of seeking treatment (Kessler et al., 2001) in individuals who initiate cocaine use in adolescence.

We first quantified the effects of adolescent cocaine exposure on behavioral flexibility in an oPFC-dependent instrumental reversal task and on dendrite arbor structure in adult excitatory deep-layer oPFC neurons. Next, we aimed to evaluate whether pre-existing morphological abnormalities in the same neuron population were associated with behavioral vulnerabilities. The model we selected for this experiment was the p190rhogap+/mouse. These mice are deficient in p190RhoGAP, a principal Src substrate in the brain that regulates cell structure through interactions with the RhoA GTPase (Brouns et al., 2000, 2001). Drugnaïve p190rhogap+/- mice appear at baseline to be behaviorally unremarkable (Gourley et al., 2012, 2013b). Nonetheless, they are highly sensitive to cocaine such that a single injection elicits a sensitization-like response (Gourley et al., 2012), making them an ideal candidate model by which to isolate pre-existing structural factors associated with subsequent cocaine vulnerability.

Our findings suggest that adolescent cocaine exposure simplifies excitatory oPFC dendritic arbors. By contrast, dendrite arbors appear grossly normal in p190rhogap+/- mice and thus, do not obviously account for behavioral vulnerabilities to cocaine in these animals.

\section{MATERIALS AND METHODS SUBJECTS}

All mice were bred on a C57BL/6 background, and those used in anatomical studies expressed thy1-derived yellow fluorescent protein (YFP; Feng et al., 2000) to enable dendrite imaging. YFP-expressing p190RhoGAP-deficient mice (p190rhogap+/-) or p190rhogap+/+ littermates were also used. p190rhogap+/mice have a $\sim 32-40 \%$ reduction in $\mathrm{p} 190 \mathrm{RhoGAP}$ protein expression (Brouns et al., 2000). Mice were maintained on a 12-h light cycle (0700 on), and provided food and water ad libitum unless otherwise noted. Procedures were in accordance with the Guide for the Care and Use of Laboratory Animals and approved by the Emory and Yale University Institutional Animal Care and Use Committees, as appropriate.

\section{ADOLESCENT COCAINE EXPOSURE}

Cocaine or saline (Sigma) was administered for five consecutive days starting at postnatal day (P) $31(10 \mathrm{mg} / \mathrm{kg}$, i.p., $1 \mathrm{ml} / 100 \mathrm{~g})$. Then, mice were left undisturbed until P56, at which point they were euthanized for anatomical studies or tested in an instrumental reversal learning task.

\section{INSTRUMENTAL REVERSAL LEARNING}

Mice with a history of adolescent cocaine exposure were foodrestricted as adults to $\sim 93 \%$ of their original body weight and trained to nose poke for food reinforcement (20 mg grain-based pellets; Bioserv) using illuminated Med-Associates conditioning chambers. Training was initiated with a continuous reinforcement schedule; 30 pellets were available for responding on each of two distinct nose poke recesses located on opposite sides of a single wall within the chambers, resulting in 60 pellets/session. Sessions ended when all 60 pellets were delivered or at $135 \mathrm{~min}$. Responding on a center aperture was not reinforced. Following 7 days of training, mice were required to "reverse" their responding to this center aperture to continue to obtain reinforcement. Responding on the previously active apertures was no longer reinforced. These "reversal" sessions were $25 \mathrm{~min}$ in duration and used a variable ratio two schedule of reinforcement (Gourley et al., 2010). Responses on the active and inactive nose poke apertures were quantified, as were head entries into the magazine where pellets were delivered. One cocaine-exposed mouse consistently generated values two standard deviations above the group mean and was excluded.

\section{LOCOMOTOR MONITORING}

In 8-week-old mice, we used a within-subjects design to compare the locomotor response to cocaine between p190rhogap $+1-$ and p190rhogap $+/+$ littermates. Mice were administered cocaine $(10 \mathrm{mg} / \mathrm{kg}$, i.p., $1 \mathrm{ml} / 100 \mathrm{~g})$ for five sequential days, and then left undisturbed for 7-10 days at which point a "challenge" injection was administered (10 mg/kg, i.p., $1 \mathrm{ml} / 100 \mathrm{~g})$.

Locomotor activity was monitored using customized MedAssociates chambers equipped with 16 photobeams. Mice were first habituated to the chambers for $1 \mathrm{~h}$ following a saline injection, then cocaine was administered. Total photobeam breaks following the cocaine injection were normalized to those generated in the $30 \mathrm{~min}$ following saline injection. During the challenge session, all mice were habituated to the locomotor monitoring chambers for $1 \mathrm{~h}$ without injection, then saline was administered and mice were monitored for $30 \mathrm{~min}$, and finally, cocaine was administered, and mice were monitored for an additional $30 \mathrm{~min}$. Cocaine-elicited photobeam breaks were normalized to those following saline in order to control for conditioned locomotor activation in response to injection. This experiment served to provide evidence that p190rhogap $+/-$ mice are hyper-sensitive to cocaine, as we have previously reported (Gourley et al., 2012), but importantly, mice used for anatomical studies were cocaine-naïve because our goal was to evaluate pre-existing factors that might be associated with cocaine vulnerability.

\section{DENDRITIC ARBOR RECONSTRUCTION AND MEASUREMENT}

Mice were euthanized, and fresh brains were submerged in $4 \%$ paraformaldehyde for $48 \mathrm{~h}$, then transferred to $30 \% \mathrm{w} / \mathrm{v}$ sucrose, followed by sectioning into $50 \mu \mathrm{m}$-thick coronal sections on a microtome held at $-15^{\circ} \mathrm{C}$. These relatively thin sections allow us to image whole deep-layer neurons without background fluorescence that would otherwise obstruct reconstruction. Little is known regarding the typical morphology of these neurons. This may be because traditional Golgi impregnation can spare deep-layer oPFC (Kolb et al., 2008), and while it is conceivable that we under-count dendrites that may have been truncated along the rostro-caudal plane, neurons with clear dendritic arbor truncations were excluded from the analyses.

Neurons were imaged on a spinning disk confocal (VisiTech International, Sunderland, UK) on a Leica microscope. Z-stacks 
were collected with a $20 \times 1.4$ NA objective using a $1-\mu \mathrm{m}$ step size, sampling above and below the neuron. After imaging, we confirmed at $10 \times$ that the image was collected from the oPFC. Most images were collected from the lateral oPFC, however, the ventral subregion was also sampled. Neurons contained at least two basal dendritic arbors and a distinct intact apical dendrite, all with at least second-order branching.

Neurons were reconstructed in three dimensions by a single experimenter blind to group using Neurolucida (MBF Biosciences). Total dendritic material was measured for apical and basal arbors. To assess dendrite complexity, a 3-D version of a Sholl analysis (Wellman et al., 2007) was performed by measuring the number of dendritic intersections within $10-\mu \mathrm{m}$ concentric spheres radiating from the soma. Four to 11 neurons/mouse from the p190rhogap+/- population and four to eight neurons/mouse from the cocaine-exposed population were imaged, reconstructed, and analyzed. Group sizes were six to seven mice in the cocaine-exposed population and four to six mice in the p190rhogap+l- population.

\section{STATISTICAL ANALYSES}

For instrumental conditioning studies, responding on the active and inactive apertures and magazine head entry rates were compared by ANOVA with repeated measures and group as the independent variable. For locomotor assessments, cocaine-elicited photobeam breaks (calculated as potentiation from baseline) were compared by ANOVA with repeated measures and group as the independent variable. Locomotor counts on the challenge day were compared between groups by unpaired $t$-test.

For anatomical studies, each mouse contributed a single value - the mean of its multiple neurons - to the analyses. Dendrite lengths were compared between groups by unpaired $t$ test. Sholl intersections were compared by ANOVA with repeated measures. In the case of interactions, post hoc comparisons were generated using Tukey's $t$-tests; the results of post hoc comparisons are indicated graphically. $p<0.05$ was considered significant. In one instance, a Kolmogorov-Smirnov comparison was also applied to total dendrite length; in this case, each neuron was considered an independent sample.

\section{RESULTS}

Here we aimed to quantify the effects of adolescent cocaine exposure on oPFC dendrite morphology. We first, however, confirmed that adolescent cocaine had long-term behavioral consequences. Mice were exposed to subchronic cocaine during early adolescence, from P31 to P35 (Spear, 2000), then left undisturbed until adulthood. Cocaine exposure is thought to confer a bias toward inflexible, maladaptive decision-making strategies, so we tested mice in an instrumental "reversal learning" task that is sensitive to chronic cocaine exposure or lesions of the oPFC in adult mice (Krueger et al., 2009; Gourley et al., 2010).

Mice with a history of subchronic saline or cocaine exposure were able to acquire a nose poke response for food reinforcement as adults (interaction $F<1$; Figure 1A). Qualitatively, cocaine appeared to modestly decrease overall responding, but this effect did not reach significance [main effect $F_{(1,21)}=3.9, p=0.06$ ], and response rates were equivalent at the end of training. When the response requirement was "reversed" such that mice were required to respond on an aperture at a separate location in the conditioning chamber, mice with a history of cocaine exposure generated fewer responses [main effect of cocaine $F_{(1,20)}=4.1$, $p<0.05$; Figure 1B]. We identified no effects of cocaine on responding on the previously reinforced aperture or magazine head entry rate (both $F<1$; Figure $1 \mathrm{C}$ ). This pattern recapitulates the effects of prolonged cocaine exposure in adult mice (Krueger et al., 2009), as well as lesions of the lateral oPFC (Gourley et al., 2010).

We next analyzed the effects of adolescent cocaine exposure on dendrite structure in the adult lateral oPFC. Representative deep-layer neurons from adult mice are shown (Figure 2A). Note

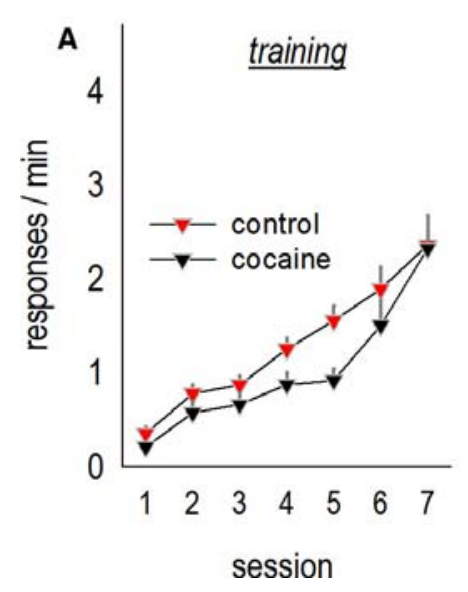

FIGURE 1 | Adolescent cocaine exposure impairs instrumental reversal learning in adulthood. (A) Mice were exposed to cocaine or saline from P31 to P35, then left undisturbed until adulthood, at which point they were trained to nose poke for food reinforcers. (B) When the location of the reinforced aperture within the chamber was then
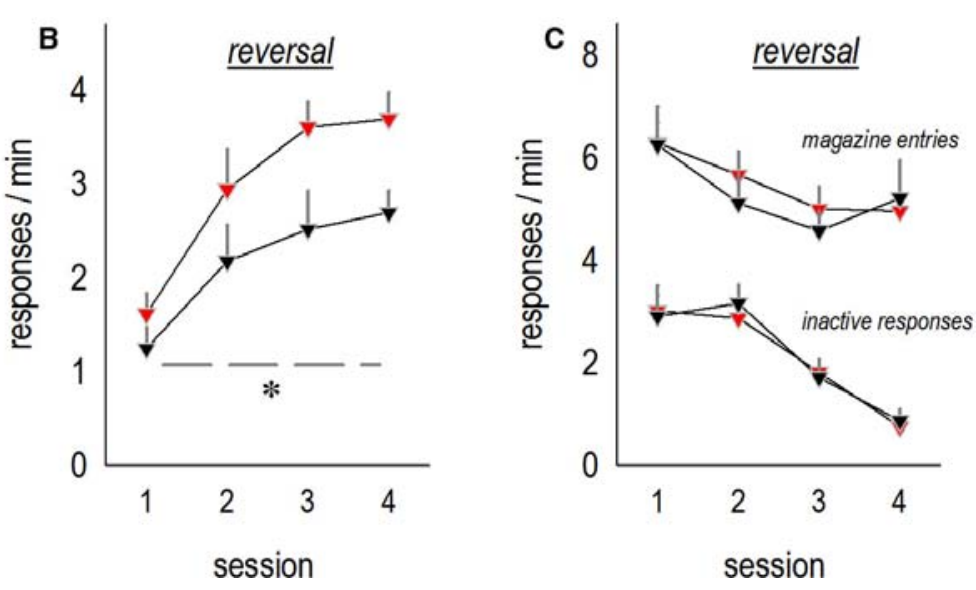

"reversed," cocaine-exposed mice generated fewer responses on the now-active aperture. (C) By contrast, cocaine exposure did not impact response inhibition on the previously reinforced apertures or head entries into the food-associated magazine. Means + SEMs, ${ }^{*} p<0.05$, main effect of cocaine. 


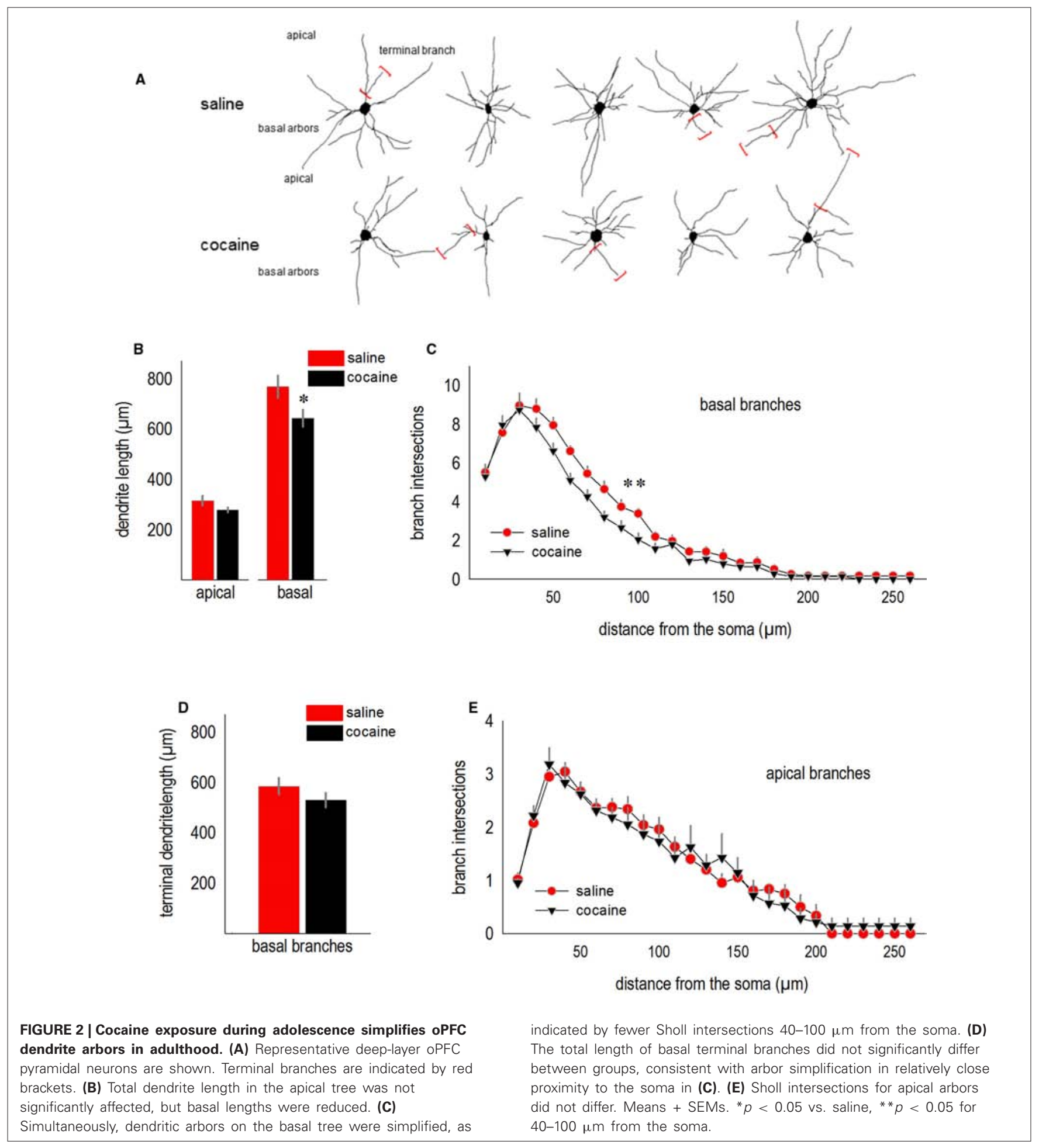

the relatively stellate shape of oPFC pyramidal neurons compared to the more classically pyramidal shape of neurons in other subregions of the prefrontal cortex (further discussed in Kolb et al., 2008; see also Liston et al., 2006; Bortolato et al., 2011).

Total basal arbor length was reduced in cocaine-exposed mice $(t 11=2.3, p<0.05$; Figure 2B). Basal arbors were also less complex, as indicated by fewer Sholl intersections 40-100 $\mu \mathrm{m}$ from the soma [interaction $F_{(25,275)}=1.9, p<0.01$; Figure $2 \mathrm{C}$ ]. As another metric of dendrite length, we measured terminal branches, the segments following the last bifurcations of each dendrite. In this case, terminal branch lengths did not differ $(t 11=-1.3, p>0.2$; Figure 2D), consistent with evidence from 
the Sholl analysis that a history of adolescent cocaine exposure simplifies dendrite arbors in close proximity to the soma (again, Figure 2C).

Despite differences in basal arbor length and complexity, apical arbors were not significantly affected (for length, $t 11=1.9, p=$ 0.09; for Sholl intersections, Fs $<1$; Figures 2B,E).

We next evaluated dendrite complexity in a developmentalgenetic model of cocaine vulnerability-p190rhogap $+/$ - mutant mice. We selected these mice because p190RhoGAP is a cytoskeleton regulatory protein implicated in postnatal dendrite stability in the brain (Sfakianos et al., 2007), and we have previously reported that $p 190$ rhogap $+/$ - mice display augmented sensitivity to cocaine (Gourley et al., 2012). Specifically, mice develop a sensitization-like response even after exposure to a single relatively low dose, and locomotor activity remains exaggerated over the course of several daily cocaine administrations, an effect that we recapitulate here by administering $10 \mathrm{mg} / \mathrm{kg}$ cocaine to mice daily for 5 days [main effect of genotype $F_{(1,20)}=7.4, p=$ 0.01 ; Figure 3A]. Mice were then left undisturbed for 1 week, after which they were habituated to the locomotor monitoring chambers for $1 \mathrm{~h}$, then injected with saline and monitored for
$30 \mathrm{~min}$, then finally, injected with cocaine. Wild type mice generated 1.4 times as many photobeam breaks following low-dose cocaine "challenge" relative to saline. By contrast, p190rhogap + /littermates broke $>4$-fold more photobeams following cocaine exposure $(t 19=-2.1, p=0.05$; Figure 3A).

Adult drug-naive p190rhogap+/- mice were crossed with mice expressing YFP, generating YFP-expressing wild type and p190rhogap+l- offspring, and allowing us to potentially identify structural predictors of cocaine vulnerable prior to drug exposure. When oPFC dendrites from drug-naïve p190rhogap+lmice were imaged and reconstructed, however, we identified no differences in total dendrite length (apical, $t 8=1.2, p=0.3$; basal, $t 8=0.8, p=0.5$; Figure $3 \mathrm{~B}$ ). By contrast, basal dendrite lengths differed in cocaine-exposed mice above, so as an additional, potentially more nuanced measure, we compared dendrite lengths using a Kolmogorov-Smirnov analysis in which the basal dendrite length from each neuron was considered an independent sample. Even here, we again did not identify differences between wild type and p190rhogap $+/-$ mice $(D=0.2, p=0.3$; Figure $3 C)$. Consistent with this outcome, basal arbor complexities did not differ, as determined by Sholl intersections (interaction $F<1$;

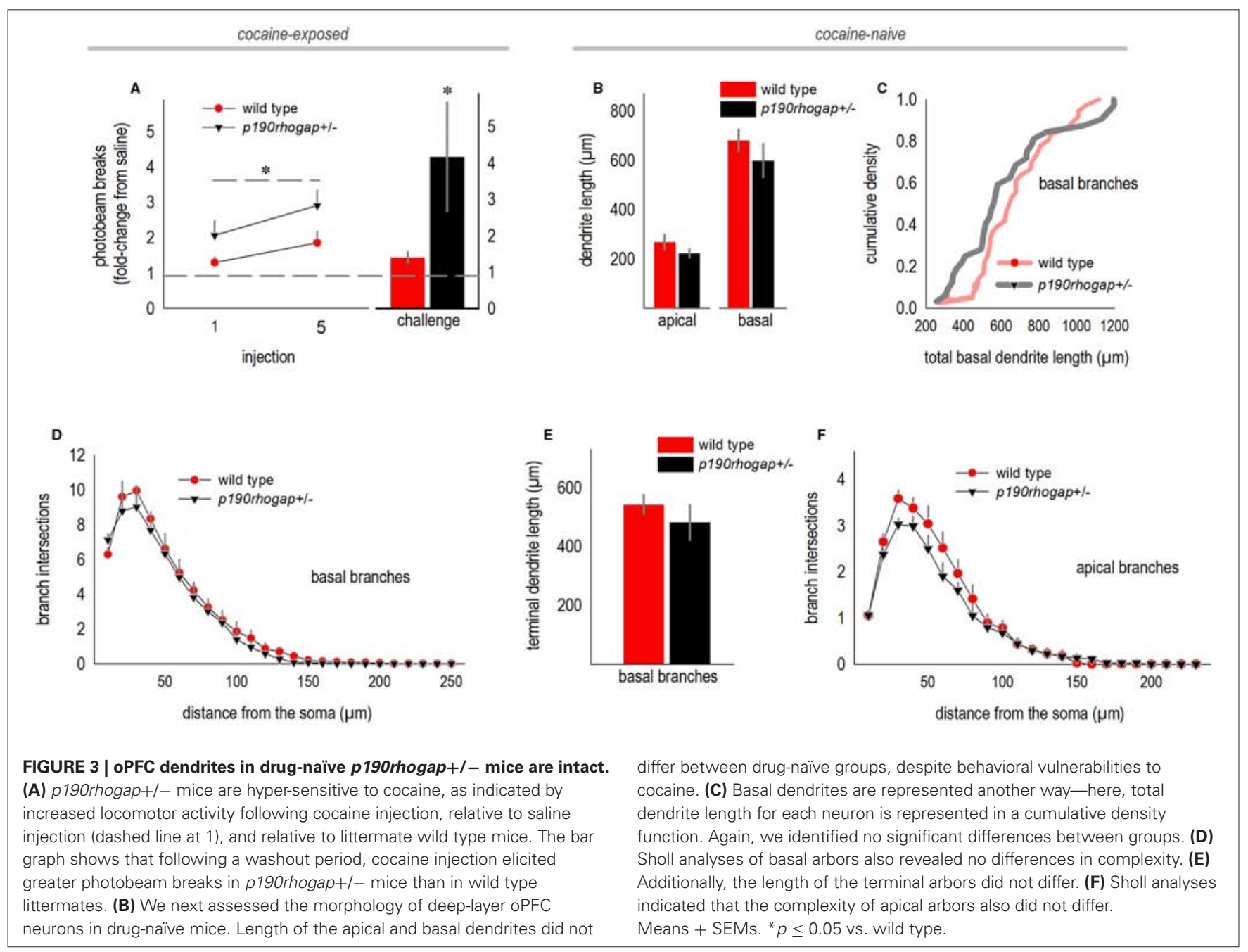


Figure 3D), and the length of the terminal branches did not differ $(t 8=-1, p=0.3$; Figure 3E).

When we quantified Sholl intersections for the apical arbor, neither interactions nor main effects were detected [interaction $F=1$; main effect $F_{(1,22)}=1.4, p=0.3$; Figure 3F]. Qualitatively, wild type mice appeared to have more complex arbors, but this impression was driven by a single mouse.

\section{DISCUSSION}

The ability of neurons to integrate into networks and regulate behavior is determined in part by the size, shape, and complexity of dendrites. Dendrites can be remarkably plastic-for example, oPFC dendritic arbors remodel following stressor exposure (Liston et al., 2006; Dias-Ferreira et al., 2009) and environmental enrichment (Comeau et al., 2010). Some such modifications may play a role in mood disorders and other psychopathologies involving cortico-striatal circuits (e.g., cocaine addiction), but the characterization of structural modifications that-like drug craving in addiction-persist beyond the period of active drug exposure remains incomplete. We used transgenic mice expressing thy1-derived YFP to isolate and reconstruct dendritic arbors of excitatory deep-layer oPFC neurons. We report that arbors remodeled in response to subchronic cocaine exposure in adolescence and were simplified in adulthood. By contrast, dendritic arbors in drug-naïve p190rhogap+/- mutant micea model of cocaine vulnerability (Gourley et al., 2012)—were intact, suggesting that the response to cocaine, rather than preexisting structural deficiencies per se, is associated with behavioral sensitivity to further drug exposure in these mice.

\section{PREFRONTAL CORTICAL DENDRITES REORGANIZE IN RESPONSE TO COCAINE}

The effects of amphetamine-like psychostimulants such as cocaine on neural structure have been intensively studied since the seminal reports of Robinson and Kolb $(1997,1999)$ describing druginduced dendrite and dendritic spine elaboration in the nucleus accumbens and medial prefrontal cortex. Within the prefrontal cortex, the vast majority of subsequent research has remained focused on medial wall structures, largely sparing the oPFC; this is despite overwhelming evidence implicating oPFC function in addiction etiology (e.g., see Lucantonio et al., 2012). Currently available data indicate that amphetamine and cocaine reduce dendritic spine density in the oPFC (Kolb et al., 2004; Crombag et al., 2005; Muhammad and Kolb, 2011 a,b; Gourley et al., 2012; but see Ferrario et al., 2005), but effects on dendrite structure remain unclear. We report novel evidence that cocaine exposure simplifies oPFC dendrite arbors, particularly in the basal region. Notably, chronic ethanol exposure does not remodel excitatory oPFC neurons (Holmes et al., 2012; DePoy et al., 2013), thus the present effects may be selective to stimulants, or potentially cocaine specifically.

In the parietal cortex, amphetamine exposure blocks the dendrite-elaborating effects of environmental enrichment, consistent with our current findings, although amphetamine alone has no consequences (Kolb et al., 2003). Nonetheless, we found evidence of long-term dendrite simplification following cocaine. How might we reconcile this apparent contradiction? One difference, in addition to the anatomical, is that cocaine was administered here during the equivalent of adolescence, a period of vulnerability to the development of dependence in humans (Anthony and Petronis, 1995; O'Brien and Anthony, 2005). Recent studies using small-animal magnetic resonance imaging complement ours, revealing that adolescent (though not adult) cocaine exposure results in cortical thinning (Wheeler et al., 2013). Interestingly, overall oPFC volume is increased following adolescent cocaine exposure; this increase could conceivably reflect glial responses to cocaine (Bowers and Kalivas, 2003; Haydon et al., 2009), though further investigations are necessary.

Here, oPFC neurons in mice with a history of adolescent cocaine exposure were simplified, particularly in the basal region. This is notable given that adolescent psychostimulant exposure in non-human primates also simplifies basal arbors in deep-layer prefrontal cortex (Selemon et al., 2007). A strong trend for a reduction in dendritic spine density was also reported by Selemon et al. (2007); similarly, oPFC dendritic spines are eliminated following adolescent cocaine exposure in the mouse (Gourley et al., 2012). The amygdala projects to deep-layer prefrontal cortex in both rodents and primates, with neurons terminating on dendritic spine heads of both apical and basal branches (Gabbott et al., 2006; Ghashghaei et al., 2007). Our findings thus indicate that the structural effects of adolescent psychostimulant exposure in critical cortico-amygdala circuits implicated in addiction (see Torregrossa et al., 2011) translate across rodent-primate species.

In tandem with arbor simplification, cocaine exposure impaired performance in an oPFC-dependent instrumental reversal task. In this task, mice are trained to nose poke for food reinforcers in a chamber with multiple response operandi. Once mice have acquired the reinforced response, the location of the reinforced aperture is "reversed," in this case, from the lateral walls of the testing chamber to a center aperture, and mice must redirect responding to this previously non-reinforced aperture. Lesions of the lateral, but not medial, oPFC delay response acquisition, as does chronic cocaine exposure in adult mice (Krueger et al., 2009; Gourley et al., 2010). Conversely, instrumental reversal learning in drug-naïve mice is associated with subsequent cocaine selfadministration patterns, with poor reversal performance predictive of higher rates of cocaine-reinforced responding (Cervantes et al., 2013). We report that even subchronic cocaine exposure in adolescent mice impaired response acquisition several weeks following drug exposure. Similarly, in a water maze reversal, early-adolescent cocaine exposure impairs response acquisition up to 10 days following exposure (Santucci et al., 2004). Together with multiple reports that cocaine exposure also occludes reversal learning based on stimulus-outcome associative contingencies (see Lucantonio et al., 2012), these findings highlight the longterm negative impact of cocaine on oPFC function, resulting in inflexible habit-like response strategies.

The oPFC also appears to regulate behavioral sensitivity and resilience to contextual stimuli associated with cocaine. For example, prolonged oPFC inactivation enhances context-induced reinstatement of cocaine seeking in rats, sparing drug-seeking behaviors induced by other conditioned stimuli (Fuchs et al., 2004; Lasseter et al., 2009). Thus, the healthy oPFC may gate the influence of contextual cues associated with drugs of abuse; repeated 
cocaine exposure could degrade this function through repeated stimulation of the dopamine D1 receptor, for example (Lasseter et al., 2014), simplification of neural structure (Figure 1), and/or imbalance between D1 and D2, given that D2 is highly expressed on basal arbors that were eliminated here (Brock et al., 1992).

\section{OPFC DENDRITE MORPHOLOGY IN DRUG-NAÏVE COCAINE-VULNERABLE MICE IS INTACT}

Structural remodeling in the central nervous system is orchestrated by Rho family GTPases including RhoA (Rho), Racl, and Cdc42, which coordinate the actin cytoskeletal rearrangements required for dendrite elaboration or simplification. Rho activation decreases branch extensions in multiple neural systems (e.g., Li et al., 2000; Wong et al., 2000), and interference with Rho activity promotes arbor growth (e.g., Sin et al., 2002; Couch et al., 2010) or activity-dependent remodeling of dendritic spines (Murakoshi et al., 2011). In the adolescent hippocampus, Rho activation causes dendritic arbor retraction, reducing overall length and complexity (Sfakianos et al., 2007).

Rho is inhibited endogenously by p190RhoGAP, which is activated by integrin receptor binding to extracellular matrix proteins (Arthur et al., 2000; Hernandez et al., 2004; Moresco et al., 2005; Bradley et al., 2006). p190rhogap-/- mice are not viable, and while p190rhogap+/- mice appear superficially normal, they exhibit significant vulnerabilities to genetic and chemical perturbations. For example, simultaneous heterozygosity for mutations in both p190RhoGAP and the cytoskeletal regulatory protein Arg kinase results in increased Rho activity and hippocampal dendritic arbor destabilization, accompanied by novel object recognition deficits (Sfakianos et al., 2007). Further, mice deficient in p190RhoGAP or the upstream effectors $\beta 1$-integrin or Arg kinase are hypervulnerable to cocaine, generating a sensitization-like response following a single injection (Gourley et al., 2009, 2012; Warren et al., 2012).

The p190rhogap+/- mouse provides an opportunity to characterize neural morphology in an organism that is behaviorally vulnerable to cocaine prior to cocaine exposure. Throughout, however, we identified no differences in the size or complexity of excitatory deep-layer oPFC neurons between mutants and p190rhogap $+/+$ littermates. These findings suggest that although cocaine exposure remodels the same neuron population, pre-existing deficiencies in dendrite arbors do not obviously account for drug vulnerability. Previously, we characterized dendritic spine density on excitatory oPFC neurons in naïve p190rhogap+l- mice and p190rhogap+/ - mice exposed to a subthreshold dose of the stress hormone corticosterone (Gourley et al., 2013b). Corticosterone reduced oPFC spine density in p190rhogap + / - mice, and these structural deficiencies emerged in concert with anhedonic-like behavior. Thus, p190RhoGAP may regulate the structural response of oPFC neurons to varied pathological insults. In line with this perspective, ethanol activates p190RhoGAP and thereby decreases actin stress fiber density in neonate astrocytes exposed to ethanol (Selva and Egea, 2011).

While we did not identify structural predictors of cocaine vulnerability in the oPFC, it is important to note that our findings do not preclude the possibility that pre-existing morphological or physiological characteristics in other models contribute to drug vulnerabilities. Additionally, pre-existing characteristics of cell populations in other brain regions-e.g., in the striatum, amygdala, or other regions of the frontal cortex (Ersche et al., 2012; Winhusen et al., 2013)—may significantly impact drug vulnerability even prior to active drug exposure.

\section{SUMMARY}

The present results contribute to the general perspective that psychostimulant-induced neural remodeling has meaningful behavioral implications. These include potentially adaptive consequences. For example, cocaine-induced dendritic spine proliferation in the nucleus accumbens has been associated with behavioral resilience (e.g., Smith et al., 2014), and blockade of certain cocaine-induced dendritic spine modifications in the oPFC and nucleus accumbens can increase — rather than occludesensitivity to subsequent cocaine exposure (Toda et al., 2006; Pulipparacharuvil et al., 2008; Gourley et al., 2012). Meanwhile, the correction of long-term or metaplastic modifications following prolonged cocaine exposure may have behavioral benefits (Shen et al., 2009; Giza et al., 2013). Animal models provide an ideal venue to disentangle these issues, and to determine neurobiological vulnerability and resiliency factors using both prospective and retrospective approaches.

\section{ACKNOWLEDGMENTS}

This work was supported by the Emory University Research Council, DA 034808, DA 036737, GM 100411, and CA 133346. The Yerkes National Primate Research Center is supported by the Office of Research Infrastructure Programs/OD P51OD11132.

\section{REFERENCES}

Anthony, J. C., and Petronis, K. R. (1995). Early-onset drug use and risk of later drug problems. Drug Alcohol Depend. 40, 9-15. doi: 10.1016/03768716(95)01194-3

Arthur, W. T., Petch, L. A., and Burridge, K. (2000). Integrin engagement suppresses RhoA activity via a c-Src-dependent mechanism. Curr. Biol. 10, 719-722. doi: 10.1016/S0960-9822(00)00537-6

Bortolato, M., Chen, K., Godar, S. C., Chen, G., Wu, W., Rebrin, I., et al. (2011). Social deficits and perseverative behaviors but not overt aggression in MAO-A hypomorphic mice. Neuropsychopharmacology 36, 2674-2688. doi: 10.1038/npp.2011.157

Bowers, M. S., and Kalivas, P. W. (2003). Forebrain astroglial plasticity is induced following withdrawal from repeated cocaine administration. Eur. J. Neurosci. 17, 1273-1278. doi: 10.1046/j.1460-9568.2003.02537.x

Bradley, W. E., Hernandez, S. E., Settleman, J., and Koleske, A. J. (2006). Integrin signaling through Arg activates p190RhoGAP by promoting its binding to p120RasGAp and recruitment to the membrane. Mol. Biol. Cell 17, 4827-4836. doi: 10.1091/mbc.E06-02-0132

Brock, J. W., Farooqui, S., Ross, K., and Prasad, C. (1992). Localization of dopamine D2 receptor protein in rat brain using polyclonal antibody. Brain Res. 578, 244 250. doi: 10.1016/0006-8993(92)90253-6

Brouns, M. R., Matheson, S. F., Hu, K. Q., Delalle, I., Caviness, V. S., Silver, J., et al. (2000). The adhesion signaling molecule p190 RhoGAP is required for morphogenetic processes in neural development. Development 127, 4891-4903. doi: $10.1038 / 35070042$

Brouns, M. R., Matheson, S. F., and Settleman, J. (2001). p190-RhoGAP is the principal Src substrate in brain and regulates axon outgrowth, guidance and fasciculation. Nat. Cell Biol. 3, 361-367. doi: 10.1038/35070042

Casey, B. J., Giedd, J. N., and Thomas, K. M. (2000). Structural and functional brain development and its relation to cognitive and development. Biol. Psychol. 54, 241-257. doi: 10.1016/S0301-0511(00)00058-2 
Cervantes, M. C., Laughlin, R. E., and Jentsch, J. D. (2013). Cocaine selfadministration behavior in inbred mouse lines segregating different capacities for inhibitory control. Psychopharmacology 229, 515-525. doi: 10.1007/s00213013-3135-4

Comeau, W. L., McDonald, R. J., and Kolb, B. E. (2010). Learning-induced alterations in prefrontal cortical dendritic morphology. Behav. Brain Res. 214, 91-101. doi: 10.1016/j.bbr.2010.04.033

Couch, B. A., DeMarco, G. J., Gourley, S. L., and Koleske, A. J. (2010). Increased dendrite branching in A $\beta P P / P S 1$ mice and elongation of dendrite arbors by fasudil administration. J. Alzheimers Dis. 20, 1003-1008. doi: 10.3233/JAD2010-091114

Crombag, H. S., Gorny, G., Li, Y., Kolb, B., and Robinson, T. E. (2005). Opposite effects of amphetamine self-administration experience on dendritic spines in the medial and orbital prefrontal cortex. Cereb. Cortex 15, 341-348. doi: 10.1093/cercor/bhh136

DePoy, L. M., Daut, R., Brigman, J. L., MacPherson, K. P., Crowley, N., Gunduz-Cinar, O., et al. (2013). Chronic alcohol produces neuroadaptations to prime striatal learning. Proc. Natl. Acad. Sci. U.S.A. 110, 14783-14788. doi: 10.1073/pnas. 1308198110

Deroche-Gamonet, V., Belin, D., and Piazza, P. V. (2004). Evidence for addictionlike behavior in the rat. Science 305, 1014-1017. doi: 10.1126/science.1099020

Dias-Ferreira, E., Sousa, J. C., Melo, I., Morqado, P., Mesquita, A. R., Cerqueria, J. J., et al. (2009). Chronic stress causes frontostriatal reorganization and affects decision-making. Science 325, 621-625. doi: 10.1126/science.1171203

Ersche, K. D., Jones, P. S., Williams, G. B., Turton, A. J., Robbins, T. W., and Bullmore, E. T. (2012). Abnormal brain structure implicated in stimulant drug addiction. Science 335, 601-604. doi: 10.1126/science. 1214463

Everitt, B. J., and Robbins, T. W. (2005). Neural systems of reinforcement for drug addiction: from actions to habits to compulsion. Nat. Neurosci. 8, 1481-1489. doi: 10.1038/nn1579

Feng, G., Mellor, R. H., Bernstein, M., Keller-Peck, C., Nguyen, Q. T., Wallace, M., et al. (2000). Imaging neuronal subsets in transgenic mice expressing multiple spectral variants of GFP. Neuron 28, 41-51. doi: 10.1016/S0896-6273(00) 00084-2

Ferrario, C. R., Gorny, G., Crombag, H. S., Li, Y., Kolb, B., and Robinson, T. E. (2005). Neural and behavioral plasticity associated with the transition from controlled to escalated cocaine use. Biol. Psychiatry 58, 751-759. doi: 10.1016/j.biopsych.2005.04.046

Fuchs, R. A., Evans, K. A., Parker, M. P., and See, R. E. (2004). Differential involvement of the orbitofrontal cortex subregions in conditioned cue-induced and cocaine-primed reinstatement of cocaine seeking in rats. J. Neurosci. 24, 6600-6610. doi: 10.1523/JNEUROSCI.1924-04.2004

Gabbott, P. L. A., Warner, T. A., and Busby, S. J. (2006). Amygdala input monosynaptically innervates parvalbumin immunoreactive local circuit neurons in rat medial prefrontal cortex. Neuroscience 139, 1039-1048. doi: 10.1016/j.neuroscience.2006.01.026

Ghashghaei, H. T., Hilgetag, C. C., and Barbas, H. (2007). Sequence of information processing for emotions based on the anatomic dialogue between pre-frontal cortex and amygdala. Neuroimage 34, 905-923. doi: 10.1016/j.neuroimage.2006.09.046

Giedd, J. N. (2004). Structural magnetic resonance imaging of the adolescent brain. Ann. N. Y. Acad. Sci. 4, 78-96. doi: 10.1196/annals.1308.009

Giza, J. I., Jung, Y., Jeffrey, R. A., Neugebauer, N. M., Picciotto, M. R., and Biederer, T. (2013). The synaptic adhesion molecule SynCAM 1 contributes to cocaine effects on synapse structure and psychostimulant behavior. Neuropsychopharmacology 38, 628-638. doi: 10.1038/npp.2012.226

Gourley, S. L., Koleske, A. J., and Taylor, J. R. (2009). Loss of dendrite stabilization by the Abl-related gene (Arg) kinase regulates behavioral flexibility and sensitivity to cocaine. Proc. Natl. Acad. Sci. U.S.A. 106, 16859-16864. doi: 10.1073/pnas.0902286106

Gourley, S. L., Lee, A. S., Howell, J. L., Piitenger, C., and Taylor, J. R. (2010). Dissociable regulation of instrumental action within mouse prefrontal cortex. Eur. J. Neurosci. 32, 1726-1734. doi: 10.1111/j.1460-9568.2010. 07438.x

Gourley, S. L., Olevska, A., Warren, M. S., Taylor, J. R., and Koleske, A. J. (2012). Arg kinase regulates prefrontal dendritic spine refinement and cocaine-induced plasticity. J. Neurosci. 32, 2314-2323. doi: 10.1523/JNEUROSCI.2730-11. 2012
Gourley, S. L., Olevska, A., Zimmermann, K. S., Ressler, K. J., DiLeone, R. J., and Taylor, J. R. (2013a). The orbitofrontal cortex regulates outcome-based decision-making via the lateral striatum. Eur. J. Neurosci. 38, 2382-2388. doi: 10.1111/ejn.12239

Gourley, S. L., Swanson, A. M., and Koleske, A. J. (2013b). Corticosteroid-induced neural remodeling predicts behavioral vulnerability and resilience. J. Neurosci. 33, 3107-3112. doi: 10.1523/JNEUROSCI.2138-12.2013

Gourley, S. L., Olevska, A., Gordon, J., and Taylor, J. R. (2013c). Cytoskeletal determinants of stimulus-response habits. J. Neurosci. 33, 11811-11816. doi: 10.1523/JNEUROSCI.1034-13.2013

Haydon, P. G., Blendy, J., Moss, S. J., and Rob Jackson, F. (2009). Astrocyte control of synaptic transmission and plasticity: a target for drugs of abuse? Neuropharmacology 56, 83-90. doi: 10.1016/j.neuropharm.2008.06.050

Hernandez, S. E., Settleman, J., and Koleske, A. J. (2004). Adhesion-dependent regulation of p190RhoGAP in the developing brain by the Abl-related gene tyrosine kinase. Curr. Biol. 14, 691-696. doi: 10.1016/j.cub.2004.03.062

Hinton, E. A., Wheeler, M. G., and Gourley, S. L. (2014). Early-life cocaine interferes with BDNF-mediated behavioral plasticity. Learn. Mem. 21, 253-257. doi: 10.1101/lm.033290.113

Holmes, A., Fitzgerald, P., Macpherson, K., DeBrouse, L., Colcaccio, G., Flynn, S. M., et al. (2012). Chronic alcohol remodels prefrontal neurons and disrupts NMDAR-mediated fear extinction encoding. Nat. Neurosci. 15, 1359-1361. doi: 10.1038/nn.3204

Jentsch, J. D., and Taylor, J. R. (1999). Impulsivity resulting from frontostriatal dysfunction in drug abuse: implications for the control of behavior by rewardrelated stimuli. Psychopharmacology 146, 373-390. doi: 10.1007/PL00005483

Kessler, R., Aguilar-Gaxiola, S., Berglund, P., Caraveo-Anduaga, J., DeWit, D., Greenfield, S., et al. (2001). Patterns and predictors of treatment seeking after onset of a substance use disorder. Arch. Gen. Psychiatry 58, 1065-1071. doi: 10.1001/archpsyc.58.11.1065

Kolb, B., Cioe, J., and Comeau, W. (2008). Contrasting effects of motor and visual spatial learning tasks on dendritic arborization and spine density in rats. Neurobiol. Learn. Mem. 90, 295-300. doi: 10.1016/j.nlm.2008.04.012

Kolb, B., Gorny, G., Li, Y., Samaha, A. N., and Robinson, T. E. (2003). Amphetamine or cocaine limits the ability of later experience to promote structural plasticity in the neocortex and nucleus accumbens. Proc. Natl. Acad. Sci. U.S.A. 100, 10523 10528. doi: 10.1073/pnas. 1834271100

Kolb, B., Pellis, S., and Robinson, T. E. (2004). Plasticity and functions of the orbital frontal cortex. Brain Cogn. 55, 104-115. doi: 10.1016/S0278-2626(03) 00278-1

Krueger, D. D., Howell, J. L., Oo, H., Olausson, P., Taylor, J. R., and Nairn, A. C. (2009). Prior chronic cocaine exposure in mice induces persistent alterations in cognitive function. Behav. Pharmacol. 20, 695-704. doi: 10.1097/FBP.0b013e328333a2bb

Lasseter, H. C., Ramirez, D. R., Xie, X., and Fuchs, R. A. (2009). Involvement of the lateral orbitofrontal cortex in drug context-induced reinstatement of cocaineseeking behavior in rats. Eur. J. Neurosci. 30, 1370-1381. doi: 10.1111/j.14609568.2009.06906.x

Lasseter, H. C., Xie, X., Arguello, A. A., Wells, A. M., Hodges, M. A., and Fuchs, R. A. (2014). Contribution of a mesocorticolimbic subcircuit to drug context-induced reinstatement of cocaine-seeking behavior in rats. Neuropsychopharmacology 39, 660-669. doi: 10.1038/npp.2013.249

Li, Z., van Aelst, L., and Cline, H. T. (2000). Rho GTPases regulate distinct aspects of dendritic growth in Xenopus central neurons in vivo. Nat. Neurosci. 3, 217 225. doi: 10.1038/72920

Liston, C., Miller, M. M., Goldwater, D. S., Radley, J. J., Rocher, A. B., Hof, P. F., et al. (2006). Stress-induced alterations in prefrontal cortical dendritic morphology predict selective impairments in perceptual attentional set-shifting. J. Neurosci. 26, 2870-7874. doi: 10.1523/JNEUROSCI.1184-06.2006

Lucantonio, F., Stalnaker, T. A., Shaham, Y., Niv, Y., and Schoenbaum, G. (2012). The impact of orbitofrontal dysfunction on cocaine addiction. Nat. Neurosci. 15, 358-366. doi: 10.1038/nn.3014

Moresco, E. M., Donaldson, S., Williamson, A., and Koleske, A. J. (2005). Integrinmediated dendrite branch maintenance requires Abelson (Abl) family kinases. J. Neurosci. 25, 6105-6118. doi: 10.1523/JNEUROSCI.1432-05.2005

Muhammad, A., and Kolb, B. (2011a). Mild prenatal stress-modulated behavior and neuronal spine density without affecting amphetamine sensitization. Dev. Neurobiol. 33, 85-98. doi: 10.1159/000324744 
Muhammad, A., and Kolb, B. (2011b). Maternal separation altered behavior and neuronal spine density without influence amphetamine sensitization. Behav. Brain Res. 223, 7-16. doi: 10.1016/j.bbr.2011.04.015

Murakoshi, H., Wang, H., and Yasuda, R. (2011). Local, persistent activation of Rho GTPases during plasticity of single dendritic spines. Nature 472, 100-104. doi: 10.1038 /nature09823

O’Brien, M. S., and Anthony, J. C. (2005). Risk of becoming cocaine dependent: epidemiological estimates for the United States, 2000-2001. Neuropsychopharmacology 30, 1006-1018. doi: 10.1038/sj.npp.1300681

Paus, T., Keshavan, M., and Giedd, J. N. (2008). Why do many psychiatric disorders emerge during adolescence? Nat. Rev. Neurosci. 9, 947-957. doi: $10.1038 / \mathrm{nrn} 2513$

Pulipparacharuvil, S., Renthal, W., Hale, C. F., Taniguchi, M., Xiao, G., Kumar, A., et al. (2008). Cocaine regulates MEF2 to control synaptic and behavioral plasticity. Neuron 59, 621-633. doi: 10.1016/j.neuron.2008. 06.020

Robinson, T. E., and Kolb, B. (1997). Persistent structural modifications in the nucleus accumbens and prefrontal cortex neurons produced by previous experience with amphetamine. J. Neurosci. 17, 8491-8497.

Robinson, T. E., and Kolb, B. (1999). Alterations in the morphology of dendrites and dendritic spines in the nucleus accumbens and prefrontal cortex following repeated treatment with amphetamine or cocaine. Eur. J. Neurosci. 11, 15981604. doi: 10.1046/j.1460-9568.1999.00576.x

Santucci, A. C., Capodilupo, S., Bernstein, J., Gomez-Ramirez, M., Milefsky, R., and Mitchell, H. (2004). Cocaine in adolescent rats produces residual memory impairments that are reversible with time. Neurotoxicol. Teratol. 26, 651-661. doi: 10.1016/j.ntt.2004.06.002

Schoenbaum, G., and Setlow, B. (2005). Cocaine makes actions insensitive to outcomes but not extinction: implications for altered orbitofrontalamygdalar function. Cereb. Cortex 15, 1162-1169. doi: 10.1093/cercor/ bhh216

Selemon, L. D., Bagovic, A., Goldman-Ranic, P. S., and Castner, S. A. (2007). Amphetamine sensitization alters dendritic morphology in prefrontal cortical pyramidal neurons in the non-human primate. Neuropsychopharmacology 32, 919-931. doi: 10.1038/sj.npp.1301179

Selva, J., and Egea, G. (2011). Ethanol increases p190RhoGAP activity, leading to actin cytoskeleton rearrangements. J. Neurochem. 119, 1306-1316. doi: 10.1111/j.1471-4159.2011.07522.x

Sfakianos, M. K., Eisman, A., Gourley, S. L., Bradley, W. D., Scheetz, A. J., Settleman, J., et al. (2007). Inhibition of Rho via Arg and p190RhoGAP in the postnatal mouse hippocampus regulates dendritic spine maturation, synapse and dendrite stability, and behavior. J. Neurosci. 27, 10982-10992. doi: 10.1523/JNEUROSCI.0793-07.2007

Shen, H. W., Toda, S., Moussawi, K., Bouknight, A., Zahm, D. S., and Kalivas, P. W. (2009). Altered dendritic spine plasticity in cocaine-withdrawn rats. J. Neurosci. 29, 2876-2884. doi: 10.1523/JNEUROSCI.5638-08.2009

Sin, W. C., Haas, K., Ruthazer, E. S., and Cline, H. T. (2002). Dendrite growth increased by visual activity required NMDA receptor and Rho GTPase. Nature 419, 475-480. doi: 10.1038/nature00987
Smith, L. N., Jedynak, J. P., Fontenot, M. R., Hale, C. F., Dietz, K. C., Taniguchi, M., et al. (2014). Fragile X mental retardation protein regulates synaptic and behavioral plasticity to repeated cocaine administration. Neuron 82, 645-658. doi: 10.1016/j.neuron.2014.03.028

Spear, L. (2000). The adolescent brain and age-related behavioral manifestations. Neurosci. Biobehav. Rev. 24, 417-463. doi: 10.1016/S0149-7634(00)00014-2

Toda, S., Shen, H. W., Peters, J., Cagle, S., and Kalivas, P. W. (2006). Cocaine increases actin cycling: effects in the reinstatement mode of drug seeking. J. Neurosci. 26, 1579-1587. doi: 10.1523/JNEUROSCI.4132-05.2006

Torregrossa, M. M., Corlett, P. R., and Taylor, J. R. (2011). Aberrant learning and memory in addiction. Neurobiol. Learn. Mem. 96, 609-623. doi: 10.1016/j.nlm.2011.02.014

Warren, M. S., Bradley, W. D., Gourley, S. L., Lin, Y. C., Reichardt, L., Greer, C. A., et al. (2012). Integrin $\beta 1$ signals through Arg to regulate postnatal dendritic arbor stability, synapse density, and behavior. J. Neurosci. 32, 2824-2834. doi: 10.1523/JNEUROSCI.3942-11.2012

Wellman, C. L., Izquierdo, A., Garret, J. E., Martin, K. P., Carroll, J., Millstein, R., et al. (2007). Impaired stress-coping and fear extinction and abnormal corticolimbic morphology in serotonin transporter knock-out mice. J. Neurosci. 27, 684-691. doi: 10.1523/JNEUROSCI.4595-06.2007

Wheeler, A. L., Lerch, J. P., Chakravarty, M. M., Friedel, M., Sled, J. G., Fletcher, P. J., et al. (2013). Adolescent cocaine exposure causes enduring macroscale changes in mouse brain structure. J. Neurosci. 33, 1797-1803. doi: 10.1523/JNEUROSCI.3830-12.2013

Winhusen, T. M., Somoza, E. C., Lewis, D. F., Kropp, F. B., Horigian, and V. E., Adinoff, B. (2013). Frontal systems deficits in stimulant-dependent patients: evidence of pre-illness dysfunction and relationship to treatment response. Drug Alcohol Depend. 127, 94-100. doi: 10.1016/j.drugalcdep.2012.06.017

Wong, W. T., Faulkner-Jones, B. E., Sanes, J. R., and Wong, R. O. (2000). Rapid dendritic remodeling in the developing retina: dependence on neurotransmission and reciprocal regulation by Rac and Rho. J. Neurosci. 20, 5024-5036.

Conflict of Interest Statement: The authors declare that the research was conducted in the absence of any commercial or financial relationships that could be construed as a potential conflict of interest.

Received: 31 July 2014; accepted: 23 September 2014; published online: 27 October 2014.

Citation: DePoy LM, Perszyk RE, Zimmermann KS, Koleske AJ and Gourley SL (2014) Adolescent cocaine exposure simplifies orbitofrontal cortical dendritic arbors. Front. Pharmacol. 5:228. doi: 10.3389/fphar.2014.00228

This article was submitted to Neuropharmacology, a section of the journal Frontiers in Pharmacology.

Copyright (c) 2014 DePoy, Perszyk, Zimmermann, Koleske and Gourley. This is an open-access article distributed under the terms of the Creative Commons Attribution License (CC BY). The use, distribution or reproduction in other forums is permitted, provided the original author(s) or licensor are credited and that the original publication in this journal is cited, in accordance with accepted academic practice. No use, distribution or reproduction is permitted which does not comply with these terms. 